Nepal Journal of Mathematical Sciences (NJMS)

ISSN: 2738-9928 (online), 2738-9812 (print)

Vol. 2, No. 2 , 2021 (August): 23-34

DOI: https://doi.org/10.3126/njmathsci.v2i2.40033

(C) School of Mathematical Sciences,

Tribhuvan University, Kathmandu, Nepal
Published Date: August 30, 2021

\title{
Performance Analysis of Service System in Health Care Network
}

\author{
Bharat Raj Wagle ${ }^{1} \&$ Ram Prasad Ghimire ${ }^{2}$ \\ ${ }^{1}$ School of Business, Pokhara University, Kaski, Nepal \\ ${ }^{2}$ Department of Mathematics, School of Science, Kathmandu University, Dhulikhel, Nepal \\ Email:1 bharatwagle@pu.edu.np, ${ }^{2}$ rpghimire@ku.edu.np
}

\begin{abstract}
This study is based on a case study research design. The approach for the study is adapted from Monte-Carlo simulation models. The study model can fit a finite number of patients who can join in the queue in different counters and performance of the selected counter in a particular time. The data are collected through direct observation with the help of a checklist. Out of 150 patients, 50 patients are observed in each new, old, and reserved (ex-army Indian pensioner and health insurance policy holder) counter. Our main findings are mean number of patients waiting in queue, mean number of patients in the system, mean time of patient waiting in queue, the time spent by a patient in the system, Average time that the server is idle, the percentage of the time that the server is busy. The study concludes that the service provided in the old counter is satisfactory to some extent. The research has been come up with the model design to estimate patients demand in the counters and it uses mean arrival time and mean service time.
\end{abstract}

Keywords: Inter-arrival time; Service time; Queueing simulation; Monte-Carlo simulation

\section{Introduction}

Study of queueing systems and their characteristics in different frameworks began from the work done by various researchers from time to time. Its history goes back to more than ten decades. A paper on the topic Waiting time and number of calls by Johannsen was published in the year 1907. It was reprinted in Occice Electrical Engineers Journal from Landon in October 1910 is assumed to be the very first paper in queueing theory. But it was found to have some mathematical errors. Thus, A. K. Erlang was the first person to study the problem of telephone networks in terms of queueing theory and he is called the father of queue $[4,13,16,21]$. Kleinrock [13] started with query how much time did you spent in waiting line in this week. It seems, we cannot escape frequent delays and they are getting progressively worse. In his book, he explain the phenomena of standing, waiting and serving, and necessity of study queueing theory. He explained global picture of where queueing system arise and why they are important. Entertaining examples are provided for attraction on reader.

Jazwinski [11] presented a unified treatment of linear and non-linear filtering theory for engineers and with sufficient emphasis on applications to enable the reader to use the theory. In attempting to fill the stated needs, the author has retained as much mathematical rigor as he felt was consistent with the prime objective to explain the theory to engineers. As a result, the author only requires of the reader background in advanced calculus, theory of ordinary differential equations and matrix analysis. Kumar [14] examined a WIMAX simulation model design with OPENET modeler 14 to measure the delay, load and the throughput performance factors. Haghighinejad [8] aimed to determine the number of patients who are waiting and waiting time in emergency department services in an Iranian hospital emergency department and to propose scenarios to reduce its queue and waiting time. For 30 days revealed that a total of 4088 patients left the emergency department after being served and 1238 patients waited in the queue for admission in the emergency department (actually these patients received services out of their defined capacity). The first scenario result in the number of beds had to be increased from 81 to 179 in order that the number waiting 
of the server become almost zero. On the other side, limit hospitalization time in the emergency department bed area to the third quartile of the serving time distribution could decrease the number waiting to 586 patients. Similarly, Qing et al. [17] studied healthcare units in many hospitals face challenges of the increased operation cost, shortage of qualified medical staff, and limited hospital facilities. Walled [1] identified unbalance between limited resources and increasing demands is the main reason leading to overcrowding in many units of hospitals.

In the field of computer engineering and design, Bhanot [7] described the supercomputer. In marine engineering Santos [20] described a probabilistic methodology they have developed to assess damaged ship survivability based on Monte-Carlo simulation. He used Monte-Carlo simulation in aerospace engineering to geometrically model in an entire spacecraft and its payload by using the integral mass model. Claude [18] introduced simulation, point wise presented advantages and disadvantages of simulation, listed future limitations to those mentioned by author. Similarly, Rarita [19] tackled a numerical package for the simulation of general queueing systems, implemented with mathematica is described on the other hand Goswami [6] studied balking and reneging in finite buffer discrete time single server queue with single and multiple working vacations. Its main theme is to avoid balking and reneging which immediately effect to optimize the revenue. Kleijnen [12] surveyed optimization of simulated systems. The survey reflects the author extensive experience with simulation of optimization through kriging (or Gaussian process) meta-models analyzed through parametric bootstrapping for deterministic and random simulation and distribution free boots trapping (or resampling) for random simulation. Alenany and Ei-Baz [3] analyzed the flow of different classes of patients into a hospital is modeled and studied by using the queueing network analyzer (QNA) algorithm and discrete event simulation. Input data for QNA are the rate and variability parameters of the arrival and service times in addition to the number of servers in each facility. Patient flows mostly match real flow for a hospital in Egypt.

Seyed [9] studied the main reliability analysis challenges in mining machinery by comparing two analytical methods and a simulation approach. In this scenario, the maintenance data from a fleet of face drilling rigs in Swedish underground metal mine were extracted by the MAXIMO system over a period of two years and were applied for analysis. This investigation reveals that the performance of these approaches in ranking and the reliability of the studies of the machines is different. However, all mentioned methods provide similar outputs but, in general, the simulation estimates the reliability of the studied machines at a higher level. Raheel [15] noted applications of simulation model for block chain system in different e-field. It described a queueing theory based model proposed for understanding the working and theoretical aspects of the block-chain. In his study he tried to validate his proposed model using the actual statistics of two popular crypto currencies, bit coin and ethereum, by running simulations for two months of transaction. Obtained performance measures parameters such as the number of transactions per block, mining time of each block, system throughput, memory pool count, waiting time in memory pool, number of unconfirmed transactions in the whole system, total number of transactions, and number of generated blocks, these values have been compared with actual statistics. Adeniran [2] deliberated single server queue system (M/M/1) which occur if arrival and service rate is Poisson distributed and Multi-server queue system which comprises of single queue many servers (M/M/c) queue with Poisson servers. Deepti [5] assumed $\mathrm{M} / \mathrm{M} / \mathrm{R}$ queue with multivariate gamma prior distribution of arrival and service and applying the Markov chain Monte-Carlo method. Huang [10] develops two models and includes eight formulas to calculate the results. They validate their model by the simulation data and verify the results by the MonteCarlo simulation method.

In this paper some essential performance measures have been obtained by using Monte-Carlo simulation. The Monte-Carlo simulation best fits in this study in the sense that arrival of the patients are random, queue formed is not systematic and patients join the queue and leave the queue haphazardly which demonstrates the queueing system confusion and no conventional queueing model formulas can handle the problem. The simulation is the technique of solving the problem by the use of data collection which cannot be solve by any conventional mathematical formulation. For this purpose the random numbers have been used under the probabilistic character of random variable. 


\section{Notation Used}

$\lambda \quad$ Arrival rate

$\mu \quad$ Service rate

$\rho \quad$ System utilization

$\mathrm{L}_{s} \quad$ Average number of customers in the system

$\mathrm{L}_{q} \quad$ Average number of customers waiting for service in the queue

$\mathrm{W}_{S} \quad$ Average time an arriving customers has to wait in the system

$\mathrm{W}_{\mathrm{q}} \quad$ Average time an arriving customers has to wait in the queue before being served

$\mathrm{P}_{0} \quad$ Probability of no customer in the system

\section{Methodology}

First ticket is provided to the first patient who comes to the counter first. Counter for coupon of new and old patient (except emergency) open from 7:00 am to 12:30 pm which we exclude in our study. There are three server for serving tickets to the patient. These three service counter provides ticket for new, old and privileged patient (Indian pensioner police, army and employees of Manipal Hospital and patient who have to take insurance policy of Nepal government). Ticket counters are open from 8:30 am to 2:00 $\mathrm{pm}$ and other service counters like as doctor's clinic, laboratory open from 9:00 am to 4:00 pm. We have collected data by which information on a phenomenon is gathered through observation. This observation involves present information of primary source. The best tool for the observations in our study is preparing checklist by direct observations. We use a cluster sampling where we took a random sample of groups or cluster of patients at Manipal Teaching Hospital between 8:30 AM to 12:30 PM at 2017-12-18 by observation of three ticket counters.

Monte-Carlo simulation is an experiment on chance so we use probability as well as random number. After getting result, we take decision under uncertainty. To understand this technique, this is break down into three steps as follows.

(i) We established cumulative distribution table.

(ii) Generate random numbers for arrival and service time distribution.

(iii) Preparing solution table that provides us certain desired results.

\section{Data Analysis and Result Discussion}

We go through a single queue system. We consider a server model with eight different inter arrival time between 1 and 31 minute. The probability of each time interval is of length corresponding inter arrival time and we use random numbers to generate customer arrival, that present in following probability distribution (P. d.) table.

Table 1: P. d. of inter arrival time of counter A

\begin{tabular}{|r|r|r|c|}
\hline Inter A. & \multicolumn{1}{|l|}{$\mathrm{P}$} & C. P. & I \\
\hline 1 & 0.38 & 0.38 & $0.00-0.37$ \\
\hline 2 & 0.24 & 0.62 & $0.38-0.61$ \\
\hline 3 & 0.22 & 0.84 & $0.62-0.83$ \\
\hline 4 & 0.06 & 0.9 & $0.84-0.89$ \\
\hline 5 & 0.04 & 0.94 & $0.90-0.93$ \\
\hline 6 & 0.02 & 0.96 & $0.94-0.95$ \\
\hline 7 & 0.02 & 0.98 & $0.96-0.97$ \\
\hline 31 & 0.02 & 1 & $0.98-0.99$ \\
\hline
\end{tabular}

Table 2: P. d. table of service time in counter A

\begin{tabular}{|c|c|c|c|}
\hline S. T. & P & C. P. & I \\
\hline 1 & 0.12 & 0.12 & $0.00-0.11$ \\
\hline 2 & 0.28 & 0.4 & $0.12-0.39$ \\
\hline 3 & 0.26 & 0.66 & $0.40-0.65$ \\
\hline 4 & 0.16 & 0.82 & $0.66-0.81$ \\
\hline 5 & 0.06 & 0.88 & $0.82-0.87$ \\
\hline 6 & 0.06 & 0.94 & $0.88-0.93$ \\
\hline 9 & 0.04 & 0.98 & $0.94-0.97$ \\
\hline 11 & 0.02 & 1 & $0.98-0.99$ \\
\hline
\end{tabular}


For service, we consider a server model with eight different service time between 1 and 11 unit minute time. The probability of each length of service time is corresponding inter arrival time (i. a. t.) and we use random numbers (r. n.) to generate customer getting service that present in following probability distribution table. Counter for coupon opened at 7:00 A. M., first patient arrived at 7:25 A. M. and has been waiting for ticket where ticket counter will opened at 8:30 A. M. Hospital counter provide ticket to patient till 12.00 noon, but here counter A was opened at 8: 35 A. M. (i.e. 5 minute later). In our study we excluded the condition of coupon counter.

Table 3 R. n. for inter arrival of server A

\begin{tabular}{|c|c|c|c|c|}
\hline 84 & 94 & 56 & 58 & 73 \\
\hline 77 & 93 & 50 & 83 & 78 \\
\hline 12 & 95 & 66 & 38 & 95 \\
\hline 43 & 20 & 4 & 34 & 59 \\
\hline 46 & 80 & 61 & 84 & 39 \\
\hline 66 & 51 & 49 & 15 & 86 \\
\hline 4 & 33 & 40 & 89 & 85 \\
\hline 75 & 24 & 10 & 99 & 42 \\
\hline 95 & 3 & 62 & 75 & 4 \\
\hline 31 & 48 & 28 & 18 & 93 \\
\hline
\end{tabular}

Table 4 R. n. for inter arrival of server A

\begin{tabular}{|c|c|c|c|c|}
\hline 91 & 9 & 8 & 4 & 87 \\
\hline 97 & 30 & 1 & 52 & 71 \\
\hline 84 & 18 & 5 & 98 & 66 \\
\hline 9 & 57 & 8 & 77 & 81 \\
\hline 90 & 2 & 5 & 43 & 77 \\
\hline 94 & 72 & 9 & 95 & 37 \\
\hline 25 & 50 & 4 & 40 & 79 \\
\hline 72 & 46 & 3 & 50 & 13 \\
\hline 76 & 24 & 5 & 9 & 96 \\
\hline 79 & 1 & 95 & 74 & 28 \\
\hline
\end{tabular}

Table 5 Solution table by Monte-Carlo simulation method of probability distribution table 1 and 2.

\begin{tabular}{|c|c|c|c|c|c|c|c|c|c|}
\hline R. N & I. A. T $\left(\boldsymbol{x}_{\mathbf{1}}\right)$ & A. T. & S. S. & R. N. & S. T. $\left(\boldsymbol{x}_{\mathbf{2}}\right)$ & S.E. & W. T. $\left(\boldsymbol{x}_{\mathbf{3}}\right)$ & S. I. T. $\left(\boldsymbol{x}_{4}\right)$ & N. W. L $\left(\boldsymbol{x}_{\mathbf{5}}\right)$ \\
\hline 84 & 4 & $8: 31$ & $8: 35$ & 91 & 6 & $8: 41$ & 4 & - & 0 \\
\hline 77 & 3 & $8: 34$ & $8: 41$ & 97 & 9 & $8: 50$ & 7 & - & 1 \\
\hline 12 & 1 & $8: 35$ & $8: 50$ & 84 & 5 & $8: 55$ & 15 & - & 1 \\
\hline 43 & 2 & $8: 37$ & $8: 55$ & 9 & 1 & $8: 56$ & 18 & - & 1 \\
\hline 46 & 2 & $8: 39$ & $8: 56$ & 90 & 6 & $9: 02$ & 17 & - & 1 \\
\hline 66 & 3 & $8: 42$ & $9: 02$ & 94 & 9 & $9: 05$ & 20 & - & 1 \\
\hline 4 & 1 & $8: 43$ & $9: 05$ & 25 & 2 & $9: 07$ & 22 & - & 1 \\
\hline 75 & 3 & $8: 46$ & $9: 07$ & 72 & 4 & $9: 11$ & 21 & - & 1 \\
\hline 95 & 6 & $8: 52$ & $9: 11$ & 76 & 4 & $9: 15$ & 19 & - & 1 \\
\hline 31 & 1 & $8: 53$ & $9: 15$ & 79 & 4 & $9: 19$ & 22 & - & 1 \\
\hline 94 & 6 & $8: 59$ & $9: 19$ & 9 & 1 & $9: 20$ & 20 & - & 1 \\
\hline 93 & 5 & $9: 04$ & $9: 20$ & 30 & 2 & $9: 22$ & 16 & - & 1 \\
\hline 95 & 6 & $9: 10$ & $9: 22$ & 18 & 2 & $9: 24$ & 12 & - & 1 \\
\hline 20 & 1 & $9: 11$ & $9: 24$ & 57 & 3 & $9: 27$ & 13 & - & 1 \\
\hline 80 & 3 & $9: 14$ & $9: 27$ & 2 & 1 & $9: 28$ & 13 & - & 1 \\
\hline 51 & 2 & $9: 16$ & $9: 28$ & 72 & 4 & $9: 32$ & 12 & - & 1 \\
\hline 33 & 1 & $9: 17$ & $9: 32$ & 50 & 3 & $9: 35$ & 15 & - & 1 \\
\hline 24 & 1 & $9: 18$ & $9: 35$ & 46 & 3 & $9: 38$ & 17 & - & 1 \\
\hline 3 & 1 & $9: 19$ & $9: 38$ & 24 & 2 & $9: 40$ & 19 & - & 1 \\
\hline 48 & 2 & $9: 21$ & $9: 40$ & 1 & 1 & $9: 41$ & 19 & - & 1 \\
\hline
\end{tabular}


Nepal Journal of Mathematical Sciences (NJMS), Vol.2, No. 2 , 2021 (August): 23-34

\begin{tabular}{|c|c|c|c|c|c|c|c|c|c|}
\hline 56 & 2 & $9: 23$ & $9: 41$ & 88 & 6 & $9: 47$ & 18 & - & 1 \\
\hline 50 & 2 & $9: 25$ & $9: 47$ & 10 & 1 & $9: 48$ & 22 & - & 1 \\
\hline 66 & 3 & $9: 28$ & $9: 48$ & 58 & 3 & $9: 51$ & 20 & - & 1 \\
\hline 4 & 1 & $9: 29$ & $9: 51$ & 83 & 5 & $9: 56$ & 22 & - & 1 \\
\hline 61 & 2 & $9: 31$ & $9: 56$ & 5 & 1 & $9: 57$ & 25 & - & 1 \\
\hline 49 & 2 & $9: 33$ & $9: 57$ & 92 & 6 & $10: 03$ & 24 & - & 1 \\
\hline 40 & 2 & $9: 35$ & $10: 03$ & 45 & 3 & $10: 06$ & 28 & - & 1 \\
\hline 10 & 1 & $9: 36$ & $10: 06$ & 30 & 2 & $10: 08$ & 30 & - & 1 \\
\hline 62 & 3 & $9: 39$ & $10: 08$ & 54 & 3 & $10: 11$ & 29 & - & 1 \\
\hline 28 & 1 & 9:40 & $10: 11$ & 95 & 9 & $10: 20$ & 31 & - & 1 \\
\hline 58 & 2 & $9: 42$ & $10: 20$ & 4 & 1 & $10: 21$ & 38 & - & 1 \\
\hline 83 & 3 & $9: 45$ & $10: 21$ & 52 & 3 & $10: 24$ & 36 & - & 1 \\
\hline 38 & 2 & $9: 47$ & $10: 24$ & 98 & 1 & $10: 25$ & 37 & - & 1 \\
\hline 34 & 1 & $9: 48$ & $10: 25$ & 77 & 4 & $10: 29$ & 37 & - & 1 \\
\hline 84 & 4 & $9: 52$ & $10: 29$ & 43 & 3 & $10: 32$ & 37 & - & 1 \\
\hline 15 & 1 & $9: 53$ & $10: 32$ & 95 & 9 & $10: 41$ & 39 & - & 1 \\
\hline 89 & 4 & $9: 57$ & $10: 41$ & 40 & 3 & $10: 44$ & 44 & - & 1 \\
\hline 99 & 31 & $10: 2$ & $10: 44$ & 50 & 3 & $10: 47$ & 16 & - & 1 \\
\hline 75 & 3 & $10: 3$ & $10: 47$ & 9 & 1 & $10: 48$ & 16 & - & 1 \\
\hline 18 & 1 & $10: 3$ & $10: 48$ & 74 & 4 & $10: 52$ & 16 & - & 1 \\
\hline 73 & 3 & $10: 3$ & $10: 52$ & 87 & 4 & $10: 56$ & 17 & - & 1 \\
\hline 78 & 3 & $10: 3$ & $10: 56$ & 71 & 4 & $11: 00$ & 18 & - & 1 \\
\hline 96 & 7 & $10: 4$ & 11:00 & 66 & 4 & $11: 04$ & 15 & - & 1 \\
\hline 59 & 2 & $10: 4$ & $11: 04$ & 81 & 4 & $11: 08$ & 17 & - & 1 \\
\hline 39 & 2 & $10: 4$ & $11: 08$ & 77 & 4 & $11: 12$ & 19 & - & 1 \\
\hline 86 & 4 & $10: 5$ & $11: 12$ & 37 & 2 & $11: 14$ & 19 & - & 1 \\
\hline 85 & 4 & $10: 5$ & $11: 14$ & 79 & 4 & $11: 18$ & 17 & - & 1 \\
\hline 42 & 2 & $10: 5$ & $11: 18$ & 13 & 2 & $11: 20$ & 19 & - & 1 \\
\hline 4 & 1 & $11: 0$ & $11: 20$ & 96 & 9 & $11: 29$ & 20 & - & 1 \\
\hline 93 & 5 & $11: 0$ & $11: 29$ & 28 & 2 & $11: 31$ & 24 & - & 1 \\
\hline & $\sum x_{1}=158$ & & & & $\sum x_{2}=182$ & & $\sum x_{3}=1070$ & $\sum x_{4}=0$ & $\sum x_{5}=49$ \\
\hline
\end{tabular}

Where S. S. = service start, S. T. = service time, S. E. = service end,

N. C. W. $=$ no. of customer waiting in line $\& \mathrm{~N}=50$.

(i) Average waiting time for a customer in queue $=\frac{\sum x_{3}}{N}=\frac{1070}{50}=21.4$ minutes

(ii) Average service time for a customer in queue $\quad=\frac{\sum x_{2}}{N}=\frac{182}{50}=3.64$ minutes

(iii) Average inter arrival time for a customer in queue $=\frac{\sum x_{1}}{N}=\frac{158}{50}=3.16$ minutes 
(iv) Average time that the server is idle $=\frac{\sum x_{4}}{N}=\frac{0}{50}=0$ minute

(v) Average number of customers waiting in queue $=\frac{\sum x_{5}}{N}=\frac{49}{50}=0.98 \sim 1$

(vi) The percentage of the time that the server is busy $=\frac{\sum x_{6}}{N} \times 100 \%=\frac{176}{205} \times 100 \%=4.89 \%$

Where $x_{6}=$ total time spent in the system, $\mathrm{N}=$ total expected time (i.e., 205 minutes).

(vii) Average time that a customer spent in the system $=\frac{\sum x_{2}}{N}+\frac{\sum x_{3}}{N}=\frac{1070}{50}+\frac{182}{50}=\frac{1252}{50}=25.04$ minutes Similarly for counter B.

Table 6 P. d. table of inter arrival time in counter B

\begin{tabular}{|r|l|l|l|}
\hline I. A. T. & P & CP & I \\
\hline 1 & 0.32 & 0.32 & $0-31$ \\
\hline 2 & 0.32 & 0.64 & $32-63$ \\
\hline 3 & 0.22 & 0.86 & $64-85$ \\
\hline 4 & 0.08 & 0.94 & $86-93$ \\
\hline 5 & 0.02 & 0.96 & $94-95$ \\
\hline 6 & 0.02 & 0.98 & $96-97$ \\
\hline 21 & 0.02 & 1 & $98-99$ \\
\hline
\end{tabular}

Table 7 P. d. table of service time in counter B

\begin{tabular}{|c|c|c|c|}
\hline S. T & P & CP & I \\
\hline 1 & 0.1 & 0.1 & $0-9$ \\
\hline 2 & 0.14 & 0.24 & $10-23$ \\
\hline 3 & 0.22 & 0.46 & $24-45$ \\
\hline 4 & 0.22 & 0.68 & $46-67$ \\
\hline 5 & 0.14 & 0.82 & $68-81$ \\
\hline 6 & 0.06 & 0.88 & $82-87$ \\
\hline 7 & 0.02 & 0.9 & $88-89$ \\
\hline 8 & 0.06 & 0.96 & $90-95$ \\
\hline 10 & 0.02 & 0.98 & $96-97$ \\
\hline 14 & 0.02 & 1 & $98-99$ \\
\hline
\end{tabular}

Counter for coupon open at 7:00 A.M. First customer arrive at 7:37 A.M. and wait for ticket where ticket counter will open at 8:34 A. M. Hospital counter provide ticket to patient till $12.30 \mathrm{PM}$. For our study our data involved only 50 patient, where patient till 12: 02PM were taken.

Table 8 R. $n$. for inter arrival of server B

\begin{tabular}{|r|r|r|r|r|}
\hline 9 & 58 & 13 & 21 & 44 \\
\hline 29 & 83 & 12 & 79 & 88 \\
\hline 64 & 65 & 70 & 33 & 22 \\
\hline 48 & 76 & 80 & 69 & 96 \\
\hline 88 & 69 & 14 & 14 & 14 \\
\hline 1 & 73 & 55 & 43 & 90 \\
\hline 7 & 79 & 50 & 6 & 31 \\
\hline 16 & 46 & 88 & 44 & 14 \\
\hline 27 & 38 & 34 & 82 & 88 \\
\hline
\end{tabular}

Table 9 R. n. of service time for server B

\begin{tabular}{|r|r|r|r|r|}
\hline 95 & 42 & 89 & 49 & 72 \\
\hline 3 & 47 & 58 & 45 & 58 \\
\hline 40 & 88 & 80 & 45 & 30 \\
\hline 5 & 26 & 32 & 96 & 73 \\
\hline 98 & 23 & 28 & 25 & 30 \\
\hline 50 & 29 & 58 & 19 & 83 \\
\hline 72 & 55 & 93 & 32 & 37 \\
\hline 91 & 5 & 39 & 38 & 49 \\
\hline 15 & 27 & 80 & 8 & 43 \\
\hline
\end{tabular}

Table 10: Solution table by Monte-Carlo simulation of probability distribution table 6 and 7

\begin{tabular}{|r|c|c|c|c|c|c|c|c|c|}
\hline R. N & I. A. T $\left(x_{1}\right)$ & A.T. & \multicolumn{1}{c|}{ S.S. } & R. N. & S. T. $\left(x_{2}\right)$ & S.E. & W. T. $\left(x_{3}\right)$ & S. I. T. $\left(x_{4}\right)$ & N. W. L $\left(x_{5}\right)$ \\
\hline 9 & 1 & $8: 34$ & $8: 35$ & 95 & 8 & $8: 43$ & 1 & - & 0 \\
\hline 29 & 1 & $8: 35$ & $8: 43$ & 3 & 1 & $8: 44$ & 8 & - & 1 \\
\hline 64 & 3 & $8: 38$ & $8: 44$ & 40 & 3 & $8: 47$ & 6 & - & 1 \\
\hline 48 & 2 & $8: 40$ & $8: 47$ & 5 & 1 & $8: 48$ & 7 & - & 1 \\
\hline 88 & 4 & $8: 44$ & $8: 48$ & 98 & 14 & $9: 02$ & 4 & - & 1 \\
\hline 1 & 1 & $8: 45$ & $9: 02$ & 50 & 4 & $9: 06$ & 17 & - & 1 \\
\hline 7 & 1 & $8: 46$ & $9: 06$ & 72 & 5 & $9: 11$ & 20 & - & 1 \\
\hline
\end{tabular}


Nepal Journal of Mathematical Sciences (NJMS), Vol.2, No. 2 , 2021 (August): 23-34

\begin{tabular}{|c|c|c|c|c|c|c|c|c|c|}
\hline 16 & 1 & $8: 47$ & $9: 11$ & 91 & 8 & $9: 19$ & 24 & - & 1 \\
\hline 27 & 1 & $8: 48$ & $9: 19$ & 15 & 2 & $9: 21$ & 31 & - & 1 \\
\hline 54 & 2 & $8: 50$ & $9: 21$ & 43 & 3 & $9: 24$ & 31 & - & 1 \\
\hline 58 & 2 & $8: 52$ & $9: 24$ & 42 & 3 & $9: 27$ & 32 & - & 1 \\
\hline 83 & 3 & $8: 55$ & $9: 27$ & 47 & 4 & $9: 31$ & 32 & - & 1 \\
\hline 65 & 3 & $8: 58$ & $9: 31$ & 88 & 7 & $9: 38$ & 33 & - & 1 \\
\hline 76 & 3 & $9: 01$ & $9: 38$ & 26 & 3 & $9: 41$ & 37 & - & 1 \\
\hline 69 & 3 & $9: 04$ & $9: 41$ & 23 & 2 & $9: 43$ & 37 & - & 1 \\
\hline 73 & 3 & $9: 07$ & $9: 43$ & 29 & 3 & $9: 46$ & 36 & - & 1 \\
\hline 79 & 3 & $9: 10$ & $9: 46$ & 55 & 4 & $9: 50$ & 36 & - & 1 \\
\hline 46 & 2 & $9: 12$ & $9: 50$ & 5 & 1 & $9: 51$ & 38 & - & 1 \\
\hline 38 & 2 & $9: 14$ & $9: 51$ & 27 & 3 & $9: 54$ & 37 & - & 1 \\
\hline 50 & 2 & $9: 16$ & $9: 54$ & 79 & 5 & $9: 59$ & 38 & - & 1 \\
\hline 13 & 1 & $9: 17$ & $9: 59$ & 89 & 7 & $10: 06$ & 42 & - & 1 \\
\hline 12 & 1 & $9: 18$ & $10: 06$ & 58 & 4 & $10: 10$ & 48 & - & 1 \\
\hline 70 & 3 & $9: 21$ & $10: 10$ & 80 & 5 & $10: 15$ & 49 & - & 1 \\
\hline 80 & 3 & $9: 24$ & $10: 15$ & 32 & 3 & $10: 18$ & 51 & - & 1 \\
\hline 14 & 1 & $9: 25$ & $10: 18$ & 28 & 3 & $10: 21$ & 53 & - & 1 \\
\hline 55 & 2 & $9: 27$ & $10: 21$ & 58 & 4 & $10: 25$ & 54 & - & 1 \\
\hline 50 & 2 & $9: 29$ & $10: 25$ & 93 & 8 & $10: 33$ & 54 & - & 1 \\
\hline 88 & 4 & $9: 33$ & $10: 33$ & 39 & 3 & $10: 36$ & 60 & - & 1 \\
\hline 34 & 2 & $9: 35$ & $10: 36$ & 80 & 5 & $10: 41$ & 61 & - & 1 \\
\hline 36 & 2 & $9: 37$ & $10: 41$ & 75 & 5 & $10: 46$ & 64 & - & 1 \\
\hline 21 & 1 & $9: 38$ & $10: 46$ & 49 & 4 & $10: 50$ & 68 & - & 1 \\
\hline 79 & 3 & $9: 41$ & $10: 50$ & 45 & 3 & $10: 53$ & 69 & - & 1 \\
\hline 33 & 2 & $9: 43$ & $10: 53$ & 45 & 3 & $10: 56$ & 70 & - & 1 \\
\hline 69 & 3 & $9: 46$ & $10: 56$ & 96 & 10 & $11: 06$ & 70 & - & 1 \\
\hline 14 & 1 & $9: 47$ & $11: 06$ & 25 & 3 & $11: 09$ & 79 & - & 1 \\
\hline 43 & 2 & $9: 49$ & 11:09 & 19 & 2 & $11: 11$ & 80 & - & 1 \\
\hline 6 & 1 & $9: 50$ & $11: 11$ & 32 & 3 & $11: 14$ & 81 & - & 1 \\
\hline 44 & 2 & $9: 52$ & $11: 14$ & 38 & 3 & $11: 17$ & 82 & - & 1 \\
\hline 82 & 4 & $9: 56$ & $11: 17$ & 8 & 1 & $11: 18$ & 81 & - & 1 \\
\hline 19 & 1 & $9: 57$ & $11: 18$ & 73 & 5 & $11: 23$ & 81 & - & 1 \\
\hline 44 & 2 & $9: 59$ & $11: 23$ & 72 & 5 & $11: 28$ & 84 & - & 1 \\
\hline 88 & 4 & $10: 03$ & $11: 28$ & 58 & 4 & $11: 32$ & 85 & - & 1 \\
\hline 22 & 1 & $10: 04$ & $11: 32$ & 30 & 3 & $11: 35$ & 88 & - & 1 \\
\hline 96 & 6 & $10: 10$ & $11: 35$ & 73 & 5 & $11: 40$ & 85 & - & 1 \\
\hline 14 & 1 & $10: 11$ & $11: 40$ & 30 & 3 & $11: 43$ & 89 & - & 1 \\
\hline 90 & 5 & $10: 16$ & $11: 43$ & 83 & 6 & $11: 49$ & 87 & - & 1 \\
\hline 31 & 1 & $10: 17$ & $11: 49$ & 37 & 3 & $11: 52$ & 92 & - & 1 \\
\hline
\end{tabular}


Bharat Raj Wagle and Ram Prasad Ghimire / Performance Analysis of Service System in Health Care Network

\begin{tabular}{|c|c|c|c|c|c|c|c|c|c|}
\hline 14 & 1 & $10: 18$ & $11: 52$ & 49 & 4 & $11: 56$ & 94 & - & 1 \\
\hline 88 & 4 & $10: 22$ & $11: 56$ & 43 & 3 & $11: 59$ & 94 & - & 1 \\
\hline 96 & 21 & $10: 43$ & $11: 59$ & 24 & 3 & $12: 02$ & 76 & - & 1 \\
\hline & $\sum x_{1}=130$ & & & & $\sum x_{2}=207$ & & $\sum x_{3}=2676$ & $\sum x_{4}=0$ & $\sum x_{5}=49$ \\
\hline
\end{tabular}

(i) Average waiting time for a customer in queue $=53.52$ minutes

(ii) Average service time for a customer in queue $=4.14$ minutes

(iii) Average inter arrival time for a customer in queue $=2.6$ minutes

(iv) Average time that the server is idle $=0$ minute

(v) Average number of customers waiting in queue $=0.98$

(vi) The percentage of the time that the server is busy $=88.08 \%$ where total time spent in the system $=$ 235 minutes \& total expected time is 235 minutes.

(vi) Average time that a customer spent in the system $=57.66$ minutes

Table 11 P. D. Table of I. Arrival Time in Counter C

\begin{tabular}{|r|c|c|c|}
\hline Inter A T & P & C. P. & I \\
\hline 1 & 0.38 & 0.38 & $00-37$ \\
\hline 2 & 0.28 & 0.66 & $38-65$ \\
\hline 3 & 0.18 & 0.84 & $66-83$ \\
\hline 4 & 0.12 & 0.96 & $84-95$ \\
\hline 6 & 0.02 & 0.98 & $96-97$ \\
\hline 20 & 0.02 & 1 & $98-99$ \\
\hline
\end{tabular}

Table 12 P. D. Table of Service Time in Counter C

\begin{tabular}{|c|c|c|c|}
\hline S.T & P & C. P. & I \\
\hline 1 & 0.72 & 0.72 & $00-71$ \\
\hline 2 & 0.14 & 0.86 & $72-85$ \\
\hline 3 & 0.08 & 0.94 & $86-93$ \\
\hline 5 & 0.04 & 0.98 & $94-97$ \\
\hline 7 & 0.02 & 1 & $98-99$ \\
\hline
\end{tabular}

Counter for coupon open at 7:00 A.M. First customer arrive at 7:25 A.M. and wait for ticket where ticket counter will open at 8:35 A. M. Hospital counter provide ticket to patient till $12.30 \mathrm{PM}$. In our data I used only 50 patient, where patient till 10: 41AM were taken.

Table 13 Random Number of Arrival for Counter C

\begin{tabular}{|r|r|r|r|r|}
\hline 59 & 12 & 67 & 53 & 82 \\
\hline 79 & 41 & 97 & 10 & 53 \\
\hline 45 & 56 & 31 & 71 & 13 \\
\hline 31 & 76 & 67 & 3 & 82 \\
\hline 42 & 33 & 31 & 41 & 15 \\
\hline 81 & 52 & 3 & 47 & 17 \\
\hline 99 & 54 & 24 & 54 & 57 \\
\hline 33 & 92 & 78 & 72 & 94 \\
\hline 89 & 3 & 3 & 79 & 79 \\
\hline 58 & 59 & 84 & 29 & 16 \\
\hline
\end{tabular}

Table 14 Random Number of Service for Counter C

\begin{tabular}{|r|r|r|r|r|}
\hline 54 & 50 & 23 & 28 & 89 \\
\hline 36 & 50 & 82 & 29 & 32 \\
\hline 34 & 58 & 87 & 60 & 85 \\
\hline 42 & 24 & 57 & 72 & 65 \\
\hline 15 & 49 & 54 & 45 & 31 \\
\hline 89 & 29 & 18 & 8 & 46 \\
\hline 18 & 31 & 86 & 15 & 28 \\
\hline 99 & 80 & 63 & 97 & 19 \\
\hline 36 & 16 & 6 & 85 & 12 \\
\hline 67 & 13 & 28 & 11 & 74 \\
\hline
\end{tabular}


Table 15 Solution Table by Monte-Carlo Simulation of Probability Distribution Table 11 and 12

\begin{tabular}{|c|c|c|c|c|c|c|c|c|c|}
\hline R. N & I. A. T $\left(x_{1}\right)$ & A.T. & S.S. & R. N. & S. T. $\left(x_{2}\right)$ & S.E. & W. T. $\left(x_{3}\right)$ & S. I. T. $\left(x_{4}\right)$ & N. W. L $\left(x_{5}\right)$ \\
\hline 59 & 2 & $8: 34$ & $8: 36$ & 54 & 1 & $8: 37$ & 2 & - & - \\
\hline 79 & 3 & $8: 37$ & $8: 37$ & 36 & 1 & $8: 38$ & - & - & - \\
\hline 45 & 2 & $8: 39$ & $8: 39$ & 34 & 1 & $8: 40$ & - & 1 & - \\
\hline 31 & 1 & $8: 40$ & $8: 40$ & 42 & 1 & $8: 41$ & - & - & - \\
\hline 42 & 2 & $8: 42$ & $8: 42$ & 15 & 1 & $8: 43$ & - & 1 & - \\
\hline 81 & 3 & $8: 45$ & $8: 45$ & 89 & 3 & $8: 48$ & - & 2 & - \\
\hline 99 & 20 & 9:05 & $9: 05$ & 18 & 1 & 9:06 & - & 17 & - \\
\hline 33 & 1 & 9:06 & 9:06 & 99 & 7 & $9: 13$ & - & - & - \\
\hline 89 & 4 & $9: 10$ & $9: 13$ & 36 & 1 & $9: 14$ & 3 & - & 1 \\
\hline 58 & 2 & $9: 12$ & $9: 14$ & 67 & 1 & $9: 15$ & 2 & - & 1 \\
\hline 12 & 1 & $9: 13$ & $9: 15$ & 50 & 1 & $9: 16$ & 2 & - & 1 \\
\hline 41 & 2 & $9: 15$ & $9: 16$ & 50 & 1 & $9: 17$ & 1 & - & 1 \\
\hline 56 & 2 & $9: 17$ & $9: 17$ & 58 & 1 & $9: 18$ & - & - & 0 \\
\hline 76 & 3 & $9: 20$ & $9: 20$ & 24 & 1 & $9: 21$ & - & 2 & - \\
\hline 33 & 1 & $9: 21$ & $9: 21$ & 49 & 1 & $9: 22$ & - & - & - \\
\hline 52 & 2 & $9: 23$ & $9: 23$ & 29 & 1 & $9: 24$ & - & 1 & - \\
\hline 54 & 2 & $9: 25$ & $9: 25$ & 31 & 1 & $9: 26$ & - & 1 & - \\
\hline 92 & 4 & $9: 29$ & $9: 29$ & 80 & 2 & $9: 31$ & - & 3 & - \\
\hline 3 & 1 & $9: 30$ & $9: 31$ & 16 & 1 & $9: 32$ & 1 & - & 1 \\
\hline 59 & 2 & $9: 32$ & $9: 32$ & 13 & 1 & $9: 33$ & - & - & - \\
\hline 67 & 3 & $9: 35$ & $9: 35$ & 23 & 1 & $9: 36$ & - & 2 & - \\
\hline 98 & 6 & $9: 41$ & $9: 41$ & 82 & 2 & $9: 43$ & - & 5 & - \\
\hline 31 & 1 & $9: 42$ & $9: 43$ & 87 & 3 & $9: 46$ & 1 & - & 1 \\
\hline 67 & 3 & $9: 45$ & $9: 46$ & 57 & 1 & $9: 47$ & 1 & - & 1 \\
\hline 31 & 1 & $9: 46$ & $9: 47$ & 54 & 1 & $9: 48$ & 1 & - & 1 \\
\hline 3 & 1 & $9: 47$ & $9: 48$ & 18 & 1 & 9:49 & 1 & - & 1 \\
\hline 24 & 1 & $9: 48$ & $9: 49$ & 86 & 3 & $9: 52$ & 1 & - & 1 \\
\hline 78 & 3 & $9: 51$ & $9: 52$ & 63 & 1 & $9: 53$ & 1 & - & 1 \\
\hline 3 & 1 & $9: 52$ & $9: 53$ & 6 & 1 & $9: 54$ & 1 & - & 1 \\
\hline 84 & 4 & $9: 56$ & $9: 56$ & 28 & 1 & $9: 57$ & - & 2 & - \\
\hline 53 & 2 & $9: 58$ & $9: 58$ & 28 & 1 & 9:59 & - & 1 & - \\
\hline 10 & 1 & $9: 59$ & $9: 59$ & 29 & 1 & 10:00 & - & - & - \\
\hline 71 & 3 & $10: 02$ & $10: 02$ & 60 & 1 & 10:03 & - & 2 & - \\
\hline 3 & 1 & $10: 03$ & $10: 03$ & 72 & 2 & 10:05 & - & - & - \\
\hline 41 & 2 & $10: 05$ & $10: 05$ & 45 & 1 & 10:06 & - & - & - \\
\hline 47 & 2 & $10: 07$ & $10: 07$ & 8 & 1 & 10:08 & - & 1 & - \\
\hline
\end{tabular}


Bharat Raj Wagle and Ram Prasad Ghimire / Performance Analysis of Service System in Health Care Network

\begin{tabular}{|c|c|c|c|c|c|c|c|c|c|}
\hline 54 & 2 & $10: 09$ & $10: 09$ & 15 & 3 & $10: 12$ & - & 1 & - \\
\hline 72 & 3 & $10: 12$ & $10: 12$ & 97 & 5 & $10: 17$ & - & - & - \\
\hline 79 & 3 & $10: 15$ & $10: 17$ & 85 & 2 & $10: 19$ & 2 & - & 1 \\
\hline 29 & 1 & $10: 16$ & $10: 19$ & 11 & 1 & $10: 20$ & 3 & - & 1 \\
\hline 82 & 3 & $10: 19$ & $10: 20$ & 89 & 3 & $10: 23$ & 1 & - & 1 \\
\hline 53 & 2 & $10: 23$ & $10: 23$ & 32 & 1 & $10: 24$ & - & - & - \\
\hline 13 & 1 & $10: 24$ & $10: 24$ & 85 & 2 & $10: 26$ & - & - & - \\
\hline 82 & 3 & $10: 27$ & $10: 27$ & 65 & 1 & $10: 28$ & - & 1 & - \\
\hline 15 & 1 & $10: 28$ & $10: 28$ & 31 & 1 & $10: 29$ & - & - & - \\
\hline 17 & 1 & $10: 29$ & $10: 29$ & 46 & 1 & $10: 30$ & - & - & - \\
\hline 57 & 2 & $10: 31$ & $10: 31$ & 28 & 1 & $10: 32$ & - & 1 & - \\
\hline 94 & 4 & $10: 35$ & $10: 35$ & 19 & 1 & $10: 36$ & - & 3 & - \\
\hline 79 & 3 & $10: 38$ & $10: 38$ & 12 & 1 & $10: 39$ & - & 2 & - \\
\hline 16 & 1 & $10: 39$ & $10: 39$ & 74 & 2 & $10: 41$ & - & - & - \\
\hline & $\sum x_{1}=125$ & & & & $\sum x_{2}=76$ & & $\sum x_{3}=24$ & $\sum x_{4}=49$ & $\sum x_{5}=15$ \\
\hline
\end{tabular}

(i) Average waiting time for a customer in queue $=0.48$ minutes

(ii) Average service time for a customer in queue $=1.52$ minutes

(iii) Average inter arrival time for a customer in queue $=2.5$ minutes

(iv) Average time that the server is idle $=0.98$ minute

(v) Average number of customers waiting in queue $=0.3$

(vi) The percentage of the time that the server is busy $=32.34 \%$ where total time spent in the system

$=76$ minutes and total expected time is 235 minutes.

(vii) Average time that a customer spent in the system $=2$ minutes

After manual calculation, we have arrival rate $\lambda=18.99,23.8,24$ and service rate $\mu=16.48,14.49,42.86$ of the Counter A, B and C respectively. The real situation of the hospital in the study of three ticket counter seemed to have $\mathrm{M} / \mathrm{M} / 1$ classical queueing model and we got performance of the queueing system by using conventional explicit formulas.

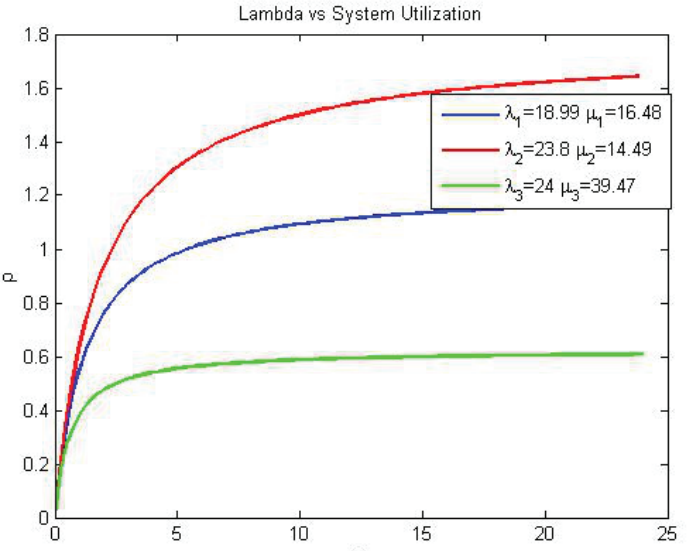

Figure 1: System Utilization vs arrival rate $(\mathrm{A}, \mathrm{B}, \mathrm{C})$

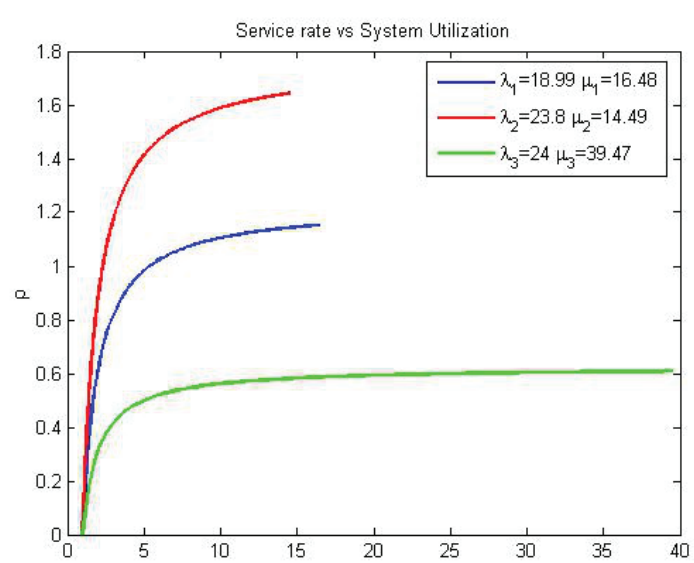

Figure 2: System Utilization vs service rate $(\mathrm{A}, \mathrm{B}, \mathrm{C})$

If system utilization $(\rho)>1$ in a queue where either the inter-arrival or service time or both are random, the queue becomes unstable, i.e., the length of the queue and the wait become infinity. If both are 
constants, $\rho>1$ implies instability. Such queues need additional servers for stability. See 1 and 2. In this study instability condition occurred in ticket counter A and B because the check list prepared by observation in rush hours. Which seems on realistic but it is due to the fact that service rate is significantly greater than arrival rate.

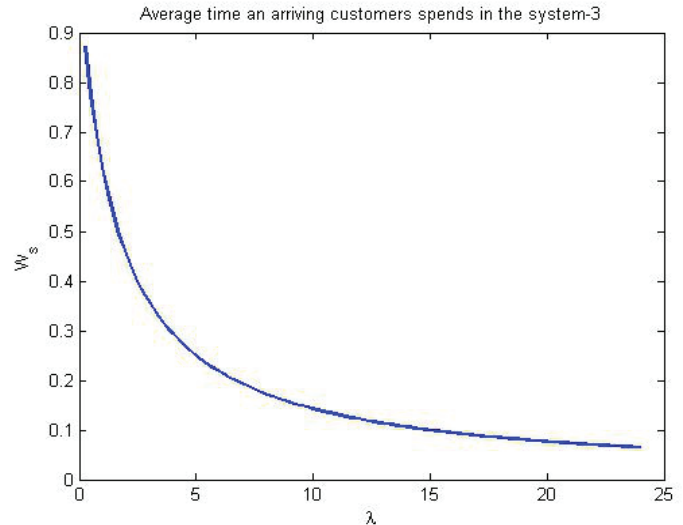

Figure 3: A. t. in system vs arrival rate (Counter 3)

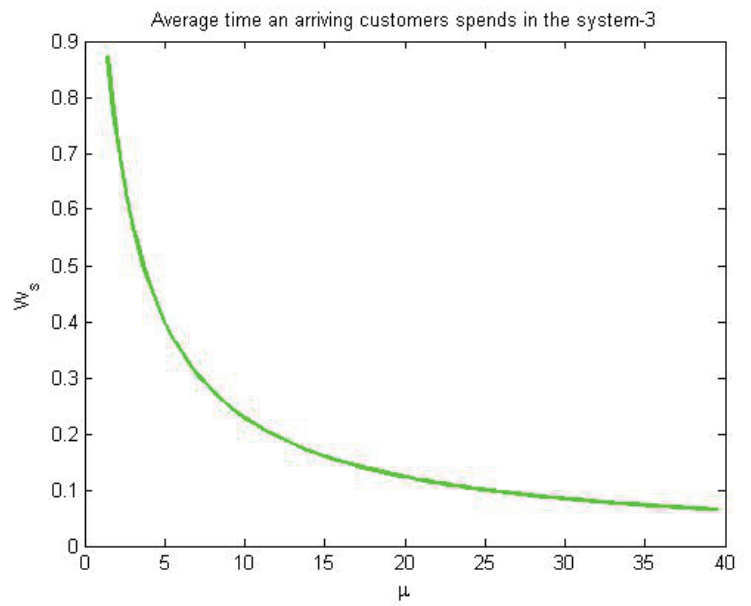

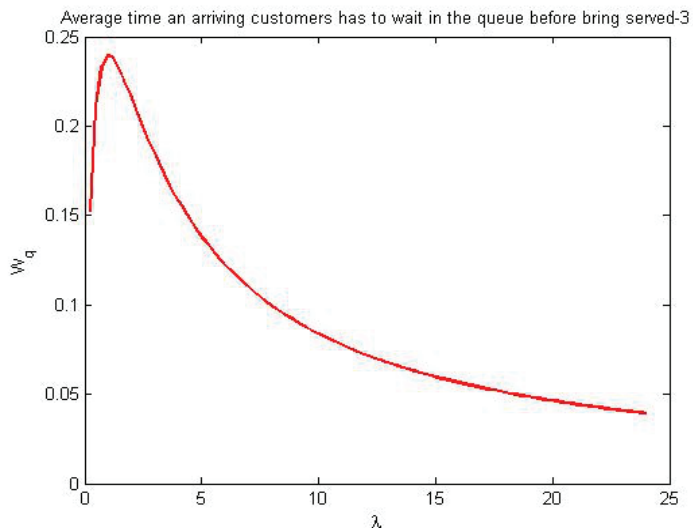

Figure 4: A. t. wait in queue vs arrival rate (counter 3)

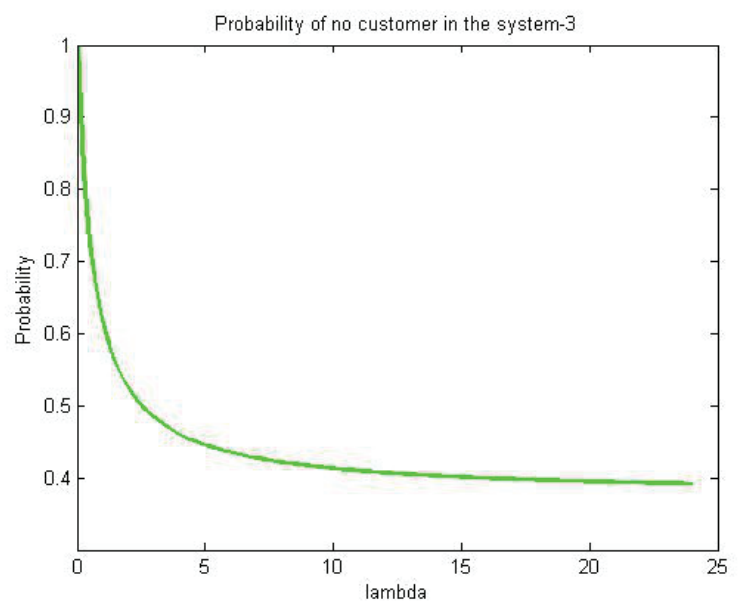

Figure 5: A. t. in system vs service rate (Counter3) Figure 6: P. of no customer vs arrival rate (counter3) From fig. 3 average time an arriving customers spend in the system in counter $\mathrm{C}$ is decreasing when arrival rate of the customers is increasing. In similar manner from fig.4 average time an arriving customers has to wait in the queue before being served initially increase up to 0.24 hours then decreasing when arrival rate increasing. There is more pressure in rush hours in counters A and B, so there should be additional server for better performance of the system.

\section{Conclusion}

We restricted our study in tickets counters of the hospitals. If we extend additional nodes (Dr. clinic, lab, pharmacy, etc.) and data of any significant days, that helps identification of the bottleneck of the system. Our study being of an exploratory and interpretive nature by using obtained numerical results. It raises a number of opportunities for future research that helps to enhance the performance of a system.

\section{References}

[1] Abo-Hamad, W. and Arisha, A. (2013). Simulation-based framework to improve patient experience in an emergency department. European Journal of Operational Research, 224 (1):154-166.

[2] Adeniran, A. O. and Kanyio, O. (2019). Quantitative model of single-server queue system. Indian 


\section{Bharat Raj Wagle and Ram Prasad Ghimire / Performance Analysis of Service System in Health Care Network}

Journal of Engineering, 16: 177-183.

[3] Alenany, E. and Ei-Baz, M. A. (2017). Modelling a hospital as a queueing network: Analysis for improving performance. World Academy of Science, Engineering and Technology, 11 (5): 1181-1187.

[4] Brockmeyer, E., Halstrom, H. L. and Jensen, A. (1948). The life of A. K. Erlang. Transactions of the Danish Academy of Technical Sciences, 2.

[5] Deepthi, V. and Jose, J. K. (2020). Bayesian estimation of an M/M/R queue with heterogeneous servers using markov chain Monte Carlo Method. Stochastic and Quality Control, 35 (2): 57-66.

[6] Goswami, V. (2014). A Discrete time queue with balking, reneging and working vacations. International Journal of Stochastic Analysis, 1-8.

[7] Gyan, B. (2005). Optimizing task layout on the blue Gene/L supercomputer. IBM Journal of Research and Development, 49: 489-500.

[8] Haghighinejad, H. A., Kharazmi, E., Hatam, N., Yousefi, S., Hesami, S. A., Danaei, M. and Askarian, M. E. (2016). Using queueing theory and simulation modeling to reduce waiting times in an Iranian emergency department. IJCBNM, 4 (1): 11-26.

[9] Hoseinie, S. H.,AI-Chalabi, H. and Ghodrati, B. (2018). Comparison between simulation and analytical method in reliability data analysis. A Case Study on Face Drilling Rigs. MDPI, 1-12.

[10] Huang, W. and Dang, J. (2020). The dynamic adjusting model of traffic queueing time-A Monte Carlo simulation study. App. Sci., 10: 63-64.

[11] Jazwinski, A. (1995). Stochastic Process and Filtering Theorey. Chapman and Hall, Singer Street.

[12] Kleijnen, J. P. C. (2017). Simulation optimization via Kriging and Bootstrapping a survey. Journal of Simulation, 8 (4): 241-250.

[13] Kleinrock, L. (1975). Queueing system. Jhoin Wiley and Sons, New York.

[14] Kumar, A. (2011). Queueing algorithm based QoS scheduling environment model in WIMAX network with OPNET modeler. Global J. of R. in Engineering Electronics and Electronics Engineering, 11 (8): 34-40.

[15] Memon, R. A., Ping, J. and Ahmed, J. (2019). Simulation model for block-chain systems using queueing theory, Eletronics, 8 (2): 1-19.

[16] Morse, P. M. (1958). Queues, Inventories and Maintenance. John Wiley and Sons, New York.

[17] Niu, Q., Peng, Q. and Elmekkawy, T. (2013). Improvement in the operating room efficiency using tabu search in simulation. Bysiness Process Management Journal, 19 (5): 799-818.

[18] Pegden, C. D.,Shannon, R. E. and Sadowski, R. P. (1995). Introduction to Simulation Using SIMAN, Mc Graw, New York.

[19] Rarita, L. and Trapel, E. (2007). Data analysis and simulation for queueing system. Department of Information Engineering and A. M., University of Salerano Fisciano Italy: Research Gate, 230-234.

[20] Santos, T. A. and Soarces, C. G. (2005). Monte-Carlo simulation of damaged ship survivability. Proceeding of the Institution of M. E. Part M-journal of Engineering for the Maritime Environment 219: 25-35.

[21] Saaty, T. L. (1999). Elements of queueing theorey with application. Dover Publications, New York. 